\title{
Morphology and motion of single optically trapped aerosol particles from digital holography
}

\section{Conference Paper}

Author(s):

David, Grégory; Esat, Kivanç (i); Thanopulos, loannis; Signorell, Ruth (1)

Publication date:

2018-09-07

Permanent link:

https://doi.org/10.3929/ethz-b-000297987

Rights / license:

In Copyright - Non-Commercial Use Permitted

Originally published in:

10723(2018), https://doi.org/10.1117/12.2321035

Funding acknowledgement:

172472 - Phase Transitions of Ultrafine Aerosol Particles: Condensation, Freezing, and Metal Formation in Confined Systems (SNF) 


\section{Morphology and motion of single optically trapped aerosol particles from digital holography}

Grégory David, Kivanç Esat, loannis Thanopulos, Ruth Signorell

Grégory David, Kivanç Esat, loannis Thanopulos, Ruth Signorell, "Morphology and motion of single optically trapped aerosol particles from digital holography," Proc. SPIE 10723, Optical Trapping and Optical Micromanipulation XV, $107231 \mathrm{~S}$ (7 September 2018); doi: $10.1117 / 12.2321035$

Event: SPIE Nanoscience + Engineering, 2018, San Diego, California, United States 


\title{
Morphology and motion of single optically trapped aerosol particles from digital holography
}

\author{
Grégory David*a ${ }^{* a}$ Kıvanç Esat ${ }^{\mathrm{a}}$, Ioannis Thanopulos ${ }^{\mathrm{b}}$, Ruth Signorell ${ }^{\mathrm{a}}$ \\ ${ }^{a}$ Department of Chemistry and Applied Biosciences, Laboratory of Physical Chemistry, ETH Zurich, \\ Vladimir-Prelog-Weg 2, CH-8093, Zurich, Switzerland; ${ }^{b}$ Department of Optics and Optometry, TEI \\ of Western Greece, Aigio 251 00, Greece
}

\begin{abstract}
Nonspherical particles play a key role in the atmosphere by affecting processes such as radiative forcing, photochemistry, new particle formation and phase transitions. In this context, measurements on single particles proved to be very useful for detailed investigations of the properties of the particles studied and of processes affecting them. However, measurements on single nonspherical particles are limited by the difficulties and lack of understanding associated with the optical trapping of such particles. Here, we aim at better understanding the optical trapping of nonspherical particles in air by comparing the motion of an observed nonspherical particle with simulated optical forces and torques. An holographic microscope is used to retrieve the 6D motion of a trapped peanut-shaped particle (3D for translation and 3D for rotation). Optical forces and torques exerted by the optical trap on the peanut-shaped particle are calculated by using FDTD simulations. Most of the main features of the particle motion are in agreement with the calculations while some specific aspects of the particle motion cannot yet be explained.
\end{abstract}

Keywords: Optical trapping, digital holography, 6D motion, optical force, optical torque, single particle, nonspherical particle, FDTD calculation.

*gregory.david@phys.chem.ethz.ch

\section{INTRODUCTION}

Aerosol particles are of great interest for both fundamental and applied research. They experience many processes including optical trapping ${ }^{1,2}$, optical forces ${ }^{3,4}$, thermophoresis ${ }^{5}$, optical binding ${ }^{6-8}$, formation of cloud droplets ${ }^{9}$, ice nucleation $^{10}$, phase transitions ${ }^{11-13}$, evaporation ${ }^{14}$, condensation of molecules ${ }^{15}$ on their surface, reactions with gases or radicals $^{16}$, photochemistry ${ }^{16,17}$, scattering and absorption of light ${ }^{18-20}$. Nonspherical aerosol particles arouse particular interest due to their specific properties. In the form of desert dust, volcanic ash, soot and ice particles they act as catalyzers of photochemical reactions ${ }^{16,17,21}$, cloud condensation nuclei ${ }^{9}$ and ice nucleation centers ${ }^{10}$. They also promote alternate pathways of new particle formation ${ }^{17}$. Due to their high loads in the atmosphere, the scattering and absorption of the sunlight by nonspherical particles also have a high impact on the Earth's radiative forcing ${ }^{22,23}$. The optical trapping of nonspherical particles and the accompanying coupling of rotation and translation still need to be better understood ${ }^{3}$. In order to unravel the role played by the particle morphology during these processes it is necessary to measure the time evolution of the aerosol particle in situ. However, very few experiments are able to perform such measurements. Usual light scattering or absorption measurements cannot simultaneously determine the particle size and shape without assuming one or the other. Conventional microscopy methods (e.g. bright field and dark field ${ }^{24}$ ) are limited because of their fixed imaging plane. This renders the study of particle dynamics virtually impossible because aerosol particles are very mobile even if they are confined in optical traps ${ }^{25}$. Furthermore, these methods can only provide two-dimensional (2D) images of the particle morphology. Three-dimensional (3D) morphology can be retrieved by imaging the particle from different angles, with Fourier ptychography ${ }^{26,27}$ and optical diffraction tomography ${ }^{28}$, or by scanning the whole particle volume with confocal imaging ${ }^{29}$. However, these methods require several measurements throughout one 3D scan. Consequently, their temporal resolution is reduced to milliseconds or seconds during which the particle must be quasi-immobile. As an alternative, digital holography (DH) provides the particle's 3D morphology ${ }^{30-32}$ and 3D position ${ }^{33}$ from each measured hologram because the reconstruction can be done at any arbitrary imaging plane $^{34}$. DH can also measure the 6D motion of nonspherical objects (3D for the position and 3D for the rotation) suspended in liquid ${ }^{33,35,36}$ or in air $^{25}$. In addition, holography is very well suited to image fast moving objects, such as Vol. 10723, 107231S · C 2018 SPIE · CCC code: 0277-786X/18/\$18 · doi: 10.1117/12.2321035 
an aerosol particle, because the imaged particle only needs to be quasi-immobile during the duration of the exposure time of each hologram, typically several $10 \mathrm{~ns}$ for pulsed lasers ${ }^{37}$ and about $10 \mu \mathrm{s}$ for continuous lasers ${ }^{38}$.

Here, a new holographic microscope is used to monitor the motion of optically-trapped nonspherical aerosol particles. The trap allows us to isolate a single particle or multiple particles in air and study them for up to several days. Such single particle measurements are playing an important role to better understand the processes involving aerosol particles $^{39-44}$, such as those mentioned above. In this paper, we present the $6 \mathrm{D}$ motion of an optically-trapped peanutshaped particle. The motion of the particle is compared with FDTD calculations of the optical forces and torques that the trap exerts on such a particle. This leads us to an improved understanding of which aspects of a nonspherical particle's motion we can predict from such calculations.

\section{METHODS}

\subsection{Digital holography of optically-trapped particles}

The experimental setup and procedure to reconstruct the shape of the trapped particles used in this study have already been presented elsewhere ${ }^{25}$. They will only be discussed briefly here. The experimental setup sketched in Figure 1 combines a digital in-line holographic microscope with a counter-propagating optical tweezer (CPT) ${ }^{43,45-47}$ to isolate a single aerosol particle or multiple aerosol particles in air. The CPT allows trapping of submicron-sized and nonspherical particles $^{25,48}$ over extended periods of time (up to several days). The continuous laser of the CPT (Laser Quantum, OPUS $3532 \mathrm{~nm}$, typical power: $500 \mathrm{~mW}$ ) is first expanded by a factor of four by using a two lense telescope. The expanded laser beam is then split by using a half-wave plate $(\lambda / 2)$ and a polarization beamsplitter cube (PBC) to obtain the two counter propagating beams with cross-polarized polarization and similar power. Finally, each beam is focused in the center of a trapping cell with an aspherical lens (focal length $=56.6 \mathrm{~mm}$ ). As shown in Figure 1-b, the foci of the two CPT beams are separated by several tens of micrometers to form a potential well in which the particles are trapped. The peanut-shaped particles studied here (Magsphere, PNT003UM) are generated by atomizing the aqueous solution containing them. The plume of particles is introduced into the trapping cell and a single particle is captured from the plume.

The digital in-line holographic microscope images the interference pattern formed by an incident reference beam and the light scattered by the trapped particle with a high speed camera (up to $4200 \mathrm{fps}$ at maximum resolution). A Kirchhoff-Helmholtz transform ${ }^{34}$ is then applied to this interference pattern to reconstruct the shape of the trapped particles. As explained by Garcia-Sucerquia et al. $^{34}$, an holographic imaging provides diffraction limited images of the particles if the reference beam is a narrow band $(\sim 0.1 \mathrm{~nm})$ spherical wave expanding from a point source. Here, the $406 \mathrm{~nm}$ holography laser is filtered to obtain a narrow band light source $(\sim 0.1 \mathrm{~nm})$. The narrow band $406 \mathrm{~nm}$ holography laser is coupled to a single mode (SM) fiber to obtain a Gaussian mode. The output of the SM fiber can hence be collimated with a lens and focused with an objective to form the spherical wave expanding from a point source. The focus of the holography laser is positioned 50-300 $\mu \mathrm{m}$ in front of the particle. A longer distance between the focus of the holography laser and the particle allows a larger field of view but a smaller zoom onto the particle (the particle represents a smaller portion of the full image). The holograms are measured with a high speed camera to obtain a time resolution of $240 \mu$ s (MotionXtra Os 7, 1920×1280 pixels, $9.12 \mu \mathrm{m}$ pixels, up to 4200 full frames per seconds, $19 \mu \mathrm{s}$ exposure time). The calculated lateral (in the YZ-plane; the axis system is defined in Figure 1) and depth (along X-axis) spatial resolution $^{34}$ of the holographic microscope are $0.77 \mu \mathrm{m}$ and $2.95 \mu \mathrm{m}$, respectively. The images of the trapped particles are reconstructed using the Octopus software (v2.0.0, 4deep, Halifax, Canada), which applies the KirchhoffHelmholtz transform ${ }^{34}$. The distance between the focus of the holography laser and the camera sensor $(\sim 21.5 \mathrm{~mm})$, the wavelength of the light $(406 \mathrm{~nm})$ and the pixel size of the camera $(9.12 \mu \mathrm{m})$ are the only required input parameters for the reconstruction of the particle morphology, position, size and orientation. The input parameters of the reconstruction and the chosen reconstruction distance (distance between the holography laser focus and the reconstruction plane) allow to calculate the size of the reconstructed image without calibration ${ }^{25}$. The particle position along the $\mathrm{X}$-axis corresponds to the reconstruction distance. The center of each reconstructed image corresponds to $Y=0$ and $Z=0$. The particle position along the $\mathrm{Y}$ - and Z-direction is calculated from the dimensions of the image. In addition, the holographic imaging allows to reconstruct the $2 \mathrm{D}$ shape of the particles at any distance between the focus of the holography laser and the camera. Hence three-dimensional morphology of the particles can be retrieved by reconstructing the shape of the particle at different $\mathrm{X}$-values. Nonetheless, the image of the particle has a lower depth resolution along the propagation 
axis of the holography beam (X-axis). In addition, holographic imaging allows us to retrieve both the 3D rotational and the 3D translational motion of nonspherical particles, as explained in next section.

(a)

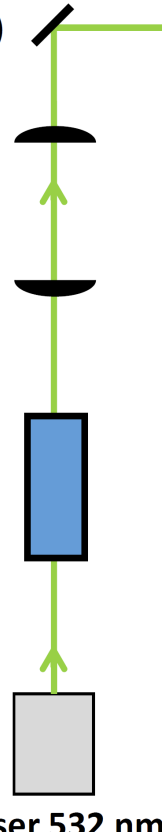

Laser $532 \mathrm{~nm}$

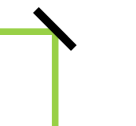

\section{CPT beam $1 \quad$ Laser $406 \mathrm{~nm}$}
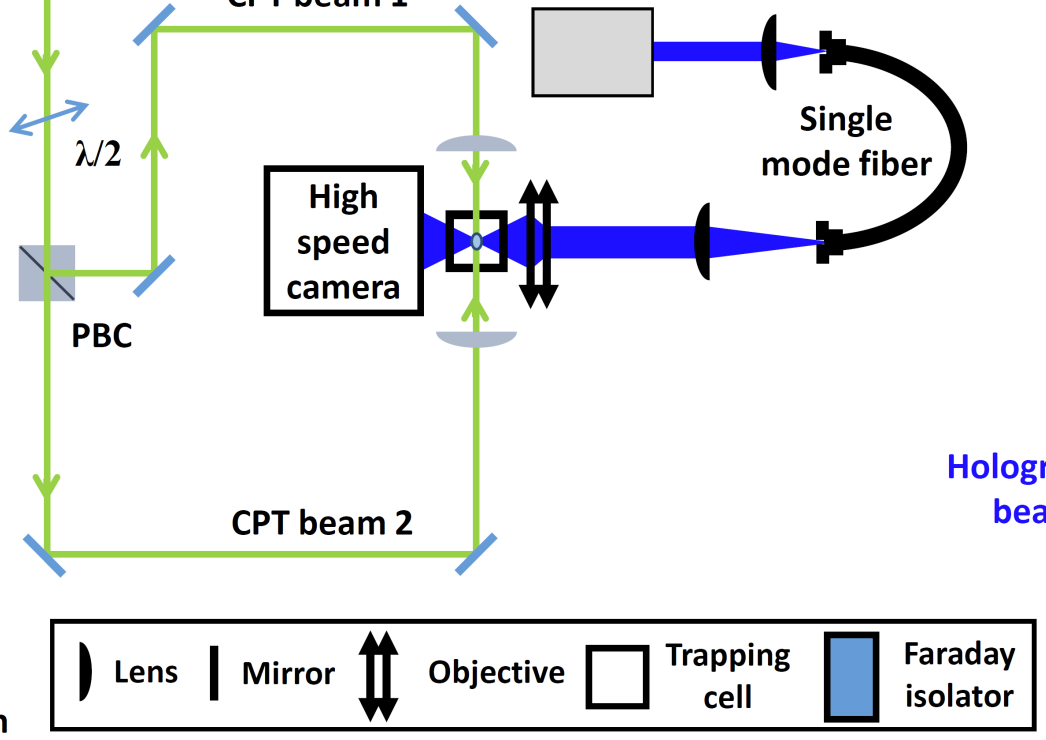

(b) CPT beam 1

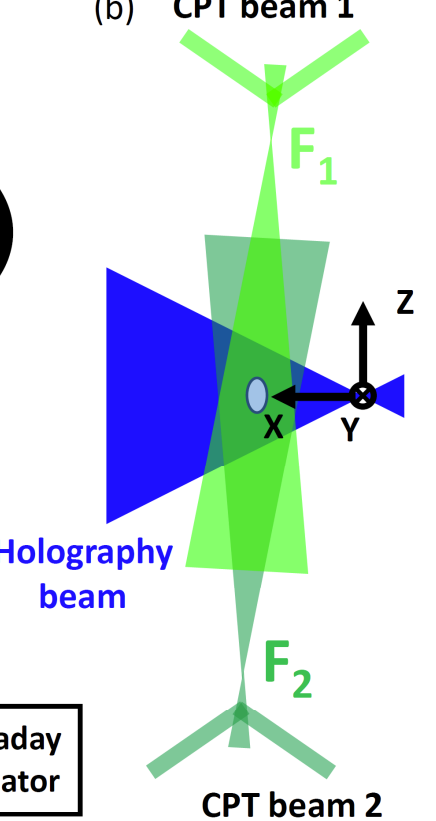

Figure 1. (a) Sketch of the experimental setup combining counter-propagating tweezers with a holographic microscope. The holography laser beam is shown in blue while the trapping beams (CPT beam1 and 2) are shown in green. (b) Zoomed-in view showing the position of the different laser beams with respect to the trapped particle (blue ellipse) and to each other. The arrow on each laser beam indicates their propagation direction. The origin of the coordinate system corresponds to the focus of the holography laser. The $\mathrm{X}$-axis corresponds to the propagation axis of the holography laser. The Z-axis and $\mathrm{Y}$-axis correspond approximately to the propagation axis of the CPT beams and the vertical direction, respectively.

\subsection{Retrieval of the orientation of a nonspherical particle}

The 3D position and 3D orientation of a nonspherical particle can be retrieved from a single hologram ${ }^{25}{ }^{35,36}$. Figure 2 illustrates how the 3D orientation of a peanut-shaped particle is retrieved by reconstructing the shape of the particle at different $X$ from the same hologram. In this example, the larger X gets the smaller (resp. higher) the intensity of the top (resp. bottom) part of the particle gets. Hence, the particle is oriented with its bottom part towards higher X-values and top part towards low $\mathrm{X}$-values. Using such procedure for time resolved measurements allows us to retrieve the $6 \mathrm{D}$ motion of a nonspherical particle (3D for translation and $3 \mathrm{D}$ for rotation) $)^{25}$. By using the reconstruction image containing the center of mass of the particle (center panel of Figure 2), the orientation of the particle within the reconstruction plane (angle $\alpha$ ) is calculated. An ellipse is fitted to the shape of the reconstructed particle to retrieve the angle $\alpha$. 

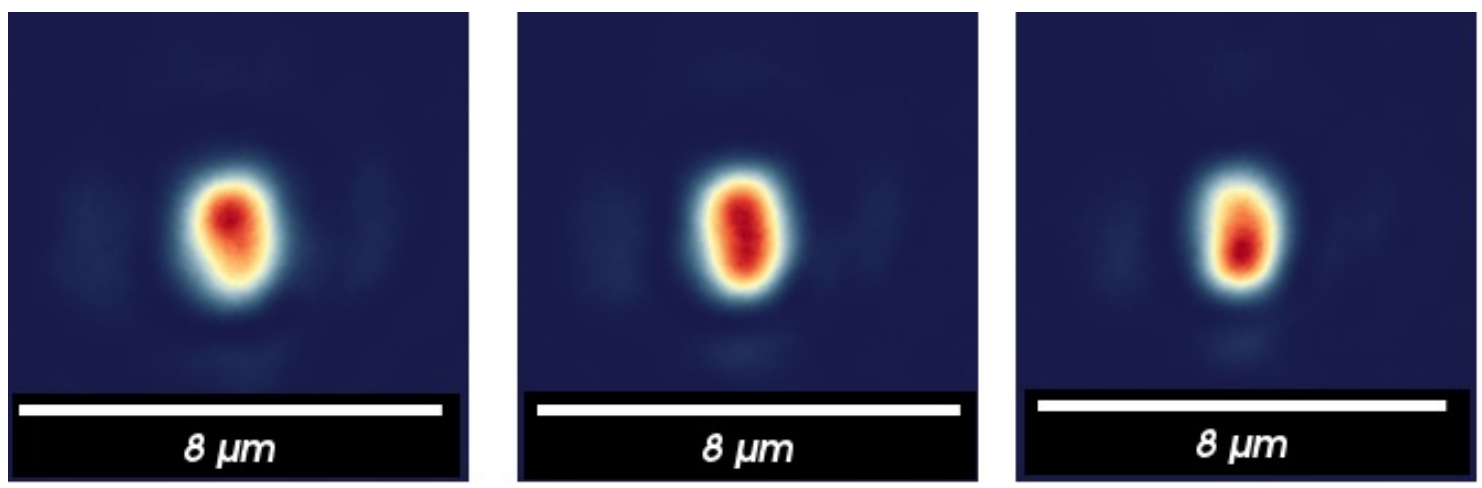

Figure 2. Reconstructed image of the particle at reconstruction distance $\mathrm{X}=111 \mu \mathrm{m}$ (left panel), $\mathrm{X}=113 \mu \mathrm{m}$ (center panel) and $\mathrm{X}=$ $115 \mu \mathrm{m}$ (right panel). The color code indicates the intensity of the reconstructed image of the particle.

The rotation angle $\gamma$ outside of the reconstruction plane (around the Y-axis) is calculated from equation (1):

$\gamma(t)=\arccos \left(s \frac{\varepsilon_{0}(t)-1}{\varepsilon_{R}-1}\right)$.

$\varepsilon_{0}(t)$ is the maximum aspect ratio of the particle in the reconstructed image recorded at time $t$, while $\varepsilon_{\mathrm{R}}$ is the aspect ratio corresponding to the long axis of the particle. The latter is determined from reconstructed images for which the long axis is aligned along the Z-direction. Both $\varepsilon_{0}(t)$ and $\varepsilon_{\mathrm{R}}$ are defined in terms of multiples of the diameter of one of the spheres that form the peanut-shaped particle. $\gamma$ is positive for counterclockwise rotations around $\mathrm{Y}$ when looking into positive $\mathrm{Y}$ direction and has values $0 \leq \gamma \leq 180^{\circ}$. Hence, $s=+1$ for $0 \leq \gamma \leq 90^{\circ}$ and $s=-1$ for $90^{\circ} \leq \gamma \leq 180^{\circ}$ in equation (1). The first derivative of the rotational angle $\alpha$ and $\gamma$ with respect to time yields the rotational velocity.

\subsection{FDTD calculations of the optical forces and torques for the peanut-shaped particle}

The optical force and torque acting on a particle are obtained by integrating Maxwell's stress tensor M over the (closed) surface $\mathrm{S}$ of the particle ${ }^{49}$. The Finite-difference time domain (FDTD) method ${ }^{50,51}$ as implemented in the FDTD Solutions package ${ }^{52}$ is used to perform these calculation as detailed in ${ }^{25}$. While in principle exact this approach becomes computationally very demanding for the particle sizes studied here. Results converged to $1 \%$ for a particle with dimensions of a few microns typically require simulation boxes extending to more than $\pm 50 \lambda$ in the directions perpendicular to the propagation axis and a grid finesse significantly better than $\lambda / 100$ close to the particle. The solution of the FDTD equations on grids of such size ( $>108$ cells including absorbing boundary conditions) typically took about $20 \mathrm{~h}$ using 16 processors sharing $256 \mathrm{~GB}$ memory. We expect all results quoted in this work to be accurate within a few percent. We note that the significant computational expense of FDTD calculations limits the density of the calculated points and thus the numerical accuracy for the case under investigation in this work.

Two sets of simulations are presented in this paper and compared with the experimentally observed motion of a peanut-shaped particle. The first set simulates the optical forces and torques exerted on a particle in the situation where the particle is closer to one trapping beam during the first part of the measurement $(\mathrm{t} \epsilon[0,48] \mathrm{ms})$ and then closer to the second beam during the second part of the data $(\mathrm{t} \in[48,77] \mathrm{ms})$, as suggested by the X-coordinate of the observed particle presented later in Figure 4. In the second set of simulations, the particle is located exactly in between both trapping beams along the $\mathrm{X}$ - and $\mathrm{Y}$-axis $(\mathrm{X}=\mathrm{Y}=0)$. In both sets the $\mathrm{Z}$-position and $\gamma$-angle of the particle are taken from the experiment (Figure 4-a and 7-a). The calculated optical forces can serve as direct indicator of the particle's velocity because the motion of such particles is overdamped ${ }^{25}$. 


\section{RESULTS}

\subsection{Retrieving rotational and translational motion of an optically-trapped nonspherical particle}

Figure 3-a shows the reconstructed images of an optically-trapped peanut-shaped particle at different times ${ }^{25}$. The time resolved image of the particle shows its translation and rotation in the optical trap. Figure 3-b shows the corresponding 3D position and orientation of the particle retrieved from the holographic imaging. The particle trajectory starts closer to the CPT beam 2 with the long axis of the particle aligned in the YZ plane. Then the particle shifts progressively toward the CPT beam 1 and rotates about the $\mathrm{Y}$-axis. The particle aligns along the $\mathrm{X}$-axis when it is approximately between the two trapping beams $(\mathrm{t}=48 \mathrm{~ms})$. As the particle get closer to the CPT beam 1, the particle keeps rotating about the $\mathrm{Y}$-axis until it is again aligned in the $\mathrm{YZ}$ plane $(\mathrm{t}=72 \mathrm{~ms})$. After $\mathrm{t}=77 \mathrm{~ms}$, the particle's rotation and translation proceed in the opposite direction (data not shown).

(a)

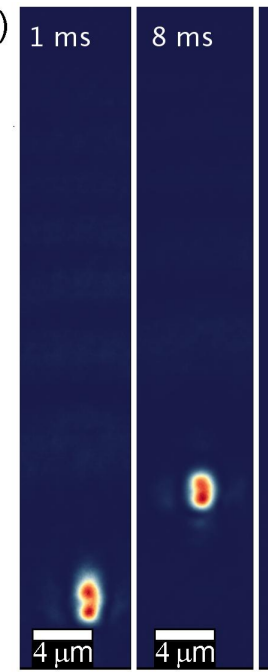

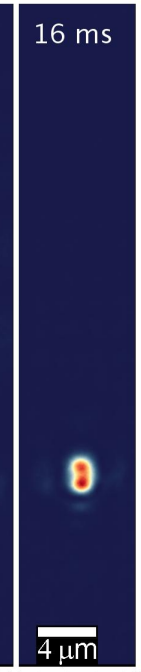
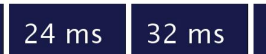

(b)
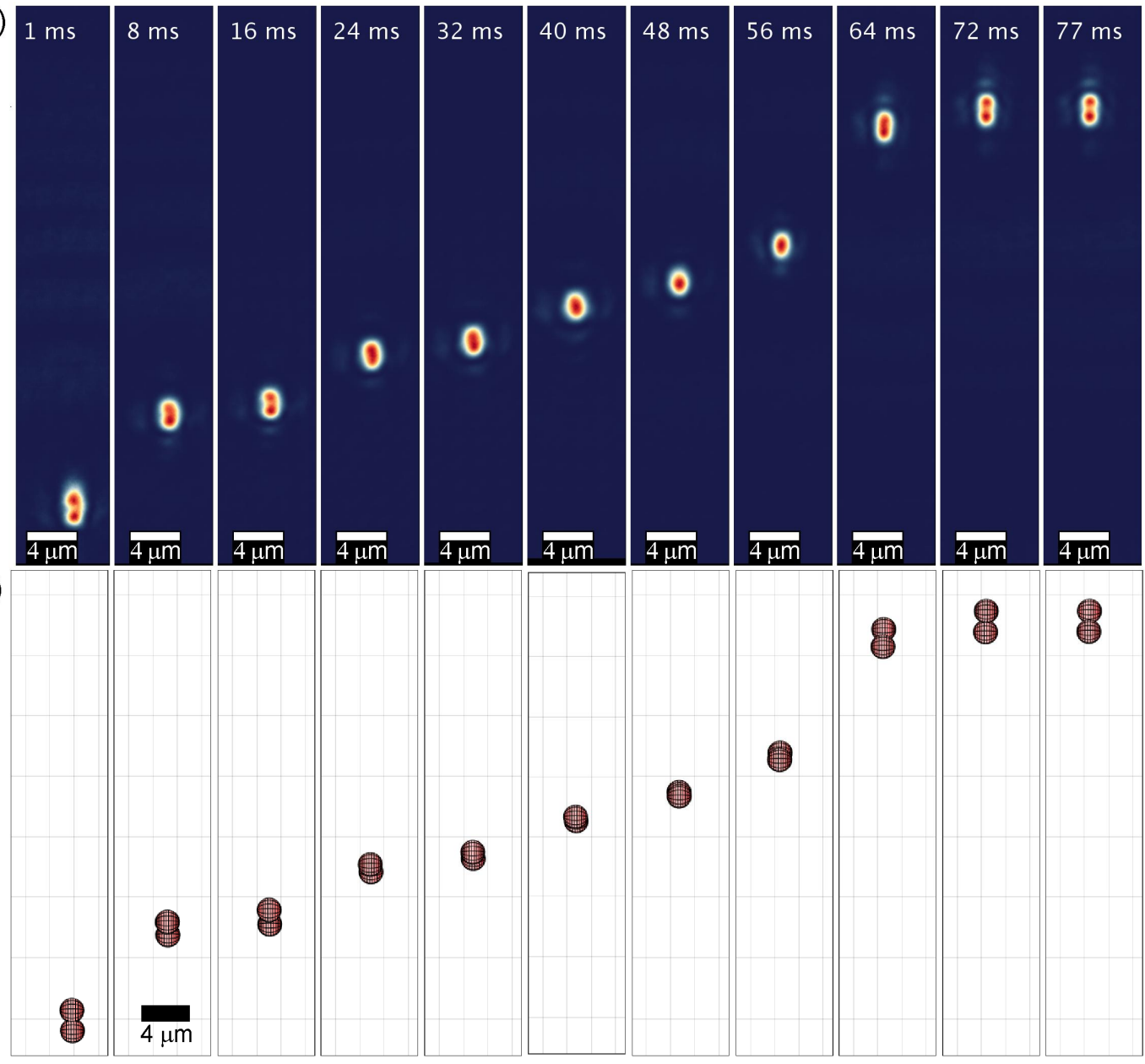

(c)
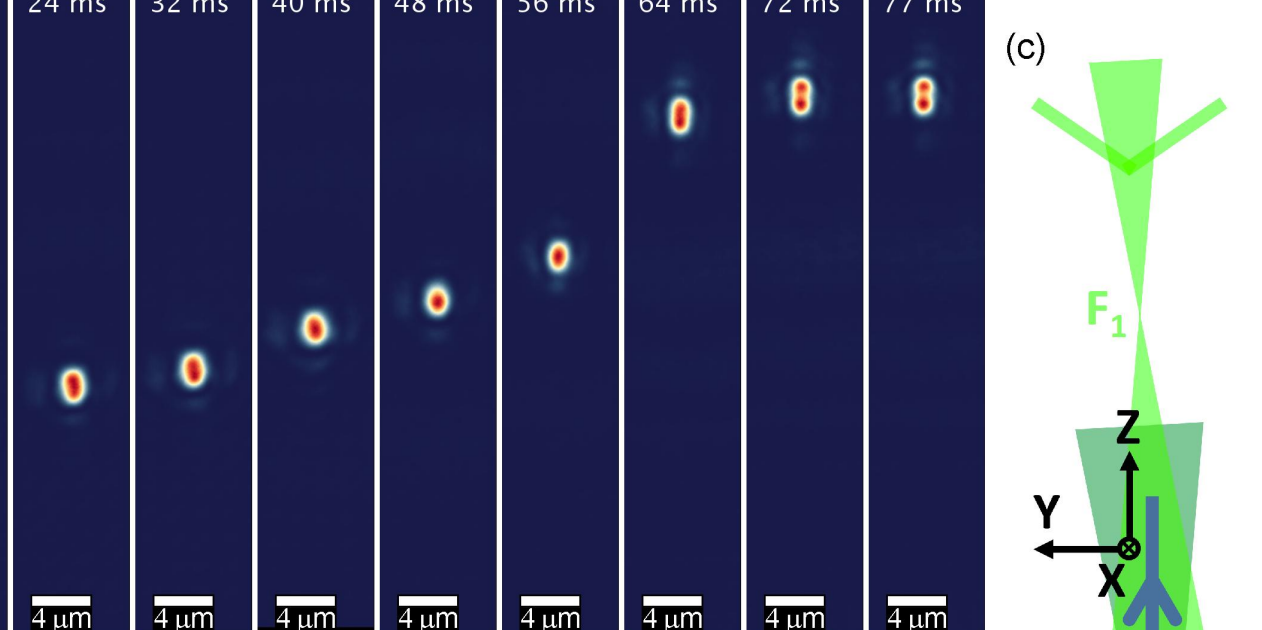

Figure 3. (a) Holographic snapshots of an optically-trapped peanut-shaped particle. (b) 3D position and orientation of the particle retrieved from each holographic snapshot. (c) Sketch of the offset CPT for the rotational and translational motion of the peanut-shaped particle in panels (a-b). The blue arrow indicates the translational motion of the particle shown in panels (a-b).

\subsection{Comparison of experimental motion with calculated forces and torque}

Figures 4-8 show the time evolution of the peanut-shaped particle's 3D position and orientation with a time resolution of $240 \mu \mathrm{s}$. The retrieved motion of the particle is compared with FDTD calculations of the optical forces and torques that the CPT trap exerts on such a particle. Figure 4-a shows the motion of the particle center of mass along the X-axis. The 
main feature of the particle motion is a rapid change of the particle position by almost $10 \mu \mathrm{m}$ around $\mathrm{t}=50 \mathrm{~ms}$. Before this change the particle is closer to the center of the CPT beam 2 and after this change the particle is closer to the center of the CPT beam 1. Figure 4-b and c present the simulated optical force along $X\left(\mathrm{~F}_{\mathrm{x}}\right)$ when the particle is located in between both trapping beams along $\mathrm{X}$ - and $\mathrm{Y}$-axis (Figure 6-a). The sign of $\mathrm{F}_{\mathrm{x}}$ indicates towards which trapping beam the peanut-shaped particle is pushed. $F_{x}$ changes from positive to negative values between $t=40 \mathrm{~ms}$ and $t=45 \mathrm{~ms}$. Hence the simulations also predict that the particle is located closer to one trapping beam during the first part of the measurement and closer to the second beam during the second part of the measurement. Quantifying the exact shift of the particle along X could not be achieved because of computation costs.
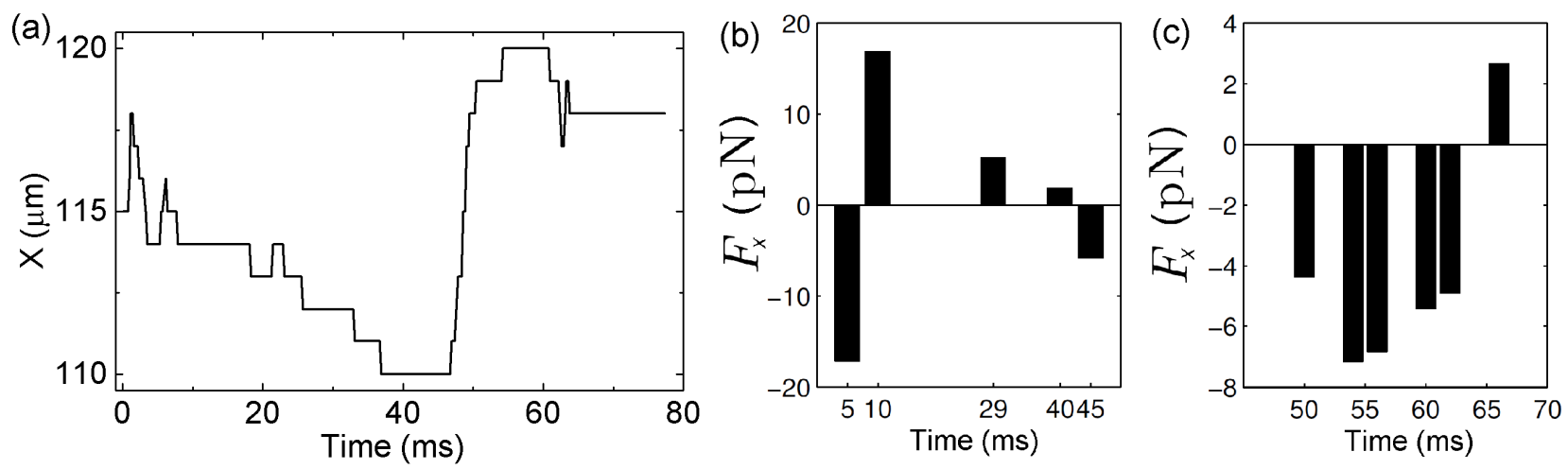

Figure 4. (a) Time evolution of the peanut-shaped particle position $X$ measured experimentally and (b-c) simulated optical force acting on the particle along the $\mathrm{X}$-axis $\left(\mathrm{F}_{\mathrm{x}}\right)$. The particle is located in between both trapping beams along $\mathrm{X}$ - and $\mathrm{Y}$-axis. The $\mathrm{Z}$ position of the particle is taken from the experiment (Figure 6-a).

Figure 5-a shows the motion of the particle along the Y-axis. The particle moves by approximately $1 \mu \mathrm{m}$ within the first $10 \mathrm{~ms}$ before moving linearly by less than $1 \mu \mathrm{m}$ during the remaining $70 \mathrm{~ms}$ of the measurement. Figure 5-b and -c present the simulated optical force along $\mathrm{Y}\left(\mathrm{F}_{\mathrm{y}}\right)$ when the particle is located in between both trapping beams along Xand $\mathrm{Y}$-axis and for the $\mathrm{Z}$ positions measured experimentally at the corresponding times. The simulated $\mathrm{F}_{\mathrm{y}}$ also predict a stronger change of the force and hence faster motion of the particle within the first $10 \mathrm{~ms}$ of the particle motion. However, the calculated $F_{y}$ does not decrease monotonously until the end and hence cannot fully explain the particle trajectory observed in the experiment. Furthermore, the calculated absolute values of $F_{x}$ and $F_{y}$ are not consistent with the amplitude of the motion of the particle along those axes. The particle moves by more than $10 \mu \mathrm{m}$ along $\mathrm{X}$ and less than $2 \mu \mathrm{m}$ along $\mathrm{Y}$ though the maximum of $\mathrm{F}_{\mathrm{x}}$ and $\mathrm{F}_{\mathrm{y}}$ are 18 and $60 \mathrm{pN}$ respectively.
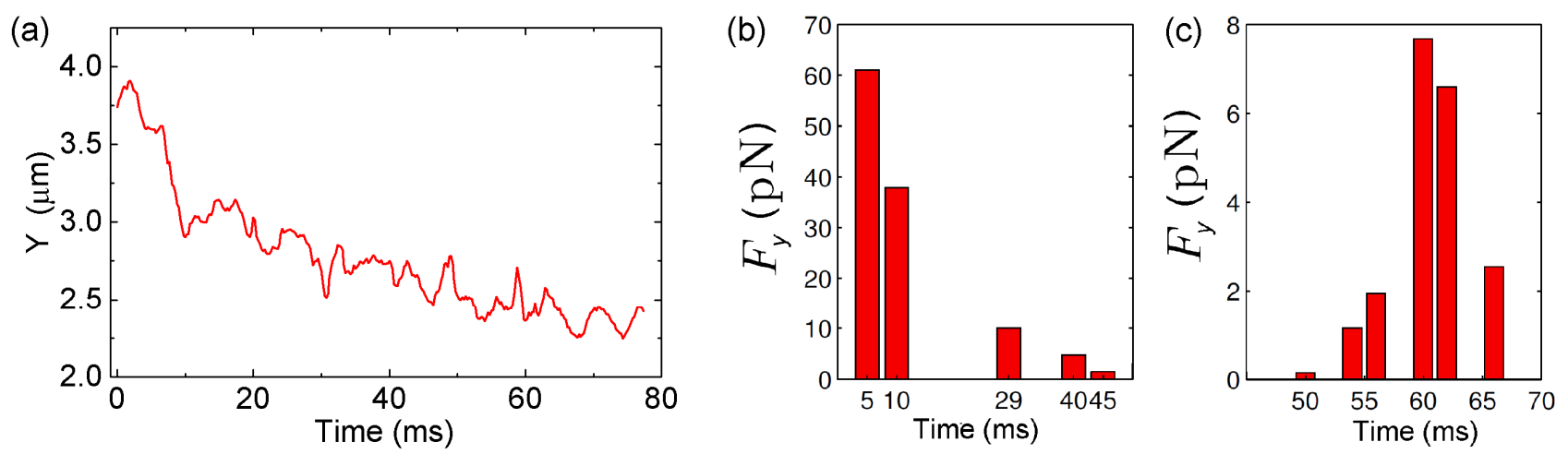

Figure 5. (a) Time evolution of the peanut-shaped particle position $Y$ measured experimentally and (b-c) simulated optical force acting on the particle along the $\mathrm{Y}$-axis $\left(\mathrm{F}_{\mathrm{y}}\right)$. The particle is located in between both trapping beams along $\mathrm{X}$ - and $\mathrm{Y}$-axis. The Zposition of the particle is taken from the experiment (Figure 6-a). 
The overall particle motion along $\mathrm{Z}$ is approximately linear from $\mathrm{t}=0$ up to $57 \mathrm{~ms}$. The particle then moves several times faster between 57 and $64 \mathrm{~ms}$. Finally the particle barely moves from 64 to to $77 \mathrm{~ms}$. At a more detailed level, the particle motion along $Z$ features periods of fast changes (e.g. $t \in[1.0,3.8] \mathrm{ms},[5.7,8.1] \mathrm{ms}$ and $[47.9,50.2] \mathrm{ms})$ and periods with almost no motion (e.g. $t \in[9.5,14.3],[26.0,30.0]$ and $[51.9,55.7] \mathrm{ms})$. The FDTD simulations of $\mathrm{F}_{\mathrm{z}}$ shown in Figure 6-b are negative until $\mathrm{t}=67 \mathrm{~ms}$. Hence, the simulations predict in agreement with the experiment that the peanut-shaped particle is continuously shifting in the same direction until $\mathrm{t}=67 \mathrm{~ms}$. However some features of the time evolution of the particle trajectory along $\mathrm{Z}$ cannot be explained by these simulations. Contrary to the experiment, the simulations are predicting a continuous decrease of the particle velocity along $Z$. While the calculated $F_{z}$ correctly predict the particle shift in one direction, they do not correctly predict the changes of velocity of the particle.
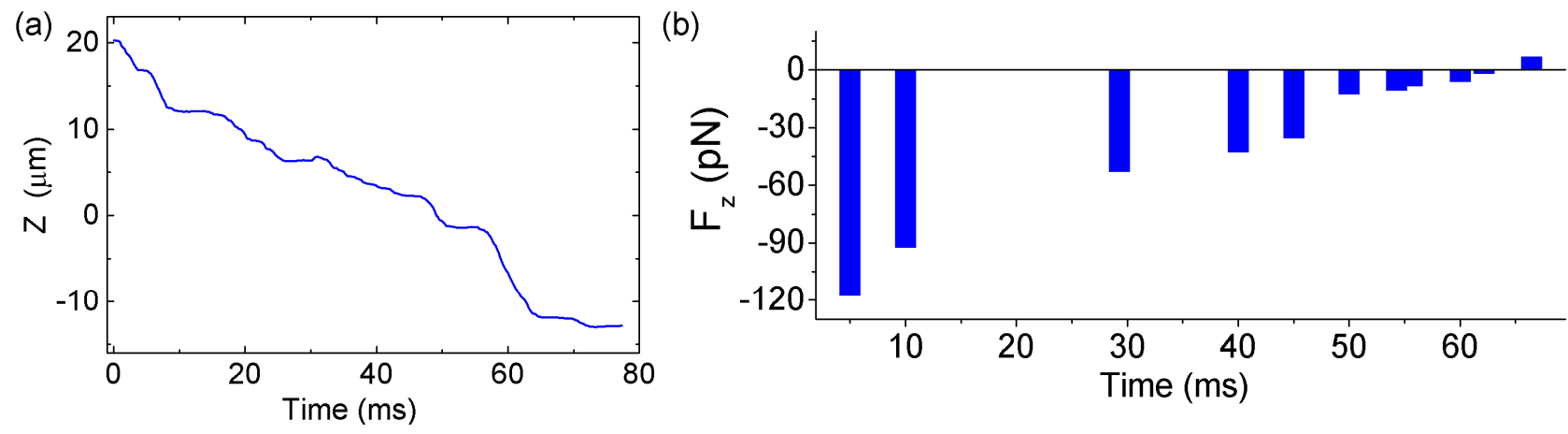

Figure 6. (a) Time evolution of the peanut-shaped particle position $\mathrm{Z}$ measured experimentally and (b) simulated optical force acting on the particle along the $\mathrm{Z}$-axis $\left(\mathrm{F}_{\mathrm{z}}\right)$. In the simulations, the particle is considered closer to one beam during the first part of the measurement $(t \in[0,48] \mathrm{ms})$ and then closer to the second beam during the second part of the data $(\mathrm{t} \epsilon[48,77] \mathrm{ms})$, as suggested by the experiment and the previous simulation.

Figure 7 and 8 present the rotation of the particle. The angle $\gamma$ measured experimentally (Figure 7 -a) is initially at $0^{\circ}$ meaning that the long axis of peanut-shaped particle lies in the reconstruction plane (YZ-plane). The particle then rotates quickly by $50^{\circ}$ within $2.5 \mathrm{~ms}$. $\gamma$ continues to increase until plateauing around $82-84^{\circ}$ at $42-47 \mathrm{~ms}$. The particle then quickly jumps to $\gamma$ angles higher than $90^{\circ}$. In other words, the orientation of the peanut-shaped particle flips, i.e. the part that initially pointed towards smaller (higher) $\mathrm{Z}$ values afterwards points toward higher (smaller) $\mathrm{Z}$-value. From 48 to $58 \mathrm{~ms}, \gamma$ plateaus again around $106-108^{\circ}$ before quickly going to $180^{\circ}$, where the particle lies again in the reconstruction plane. The simulated torque acting along the $\mathrm{Y}$-axis $\left(\mathrm{T}_{\mathrm{y}}\right)$ is consistent with the observed $\gamma$-values. $\mathrm{T}_{\mathrm{y}}$ is relatively small from 20 to $55 \mathrm{~ms}$, but larger within the first ten milliseconds and around 60-65 ms.
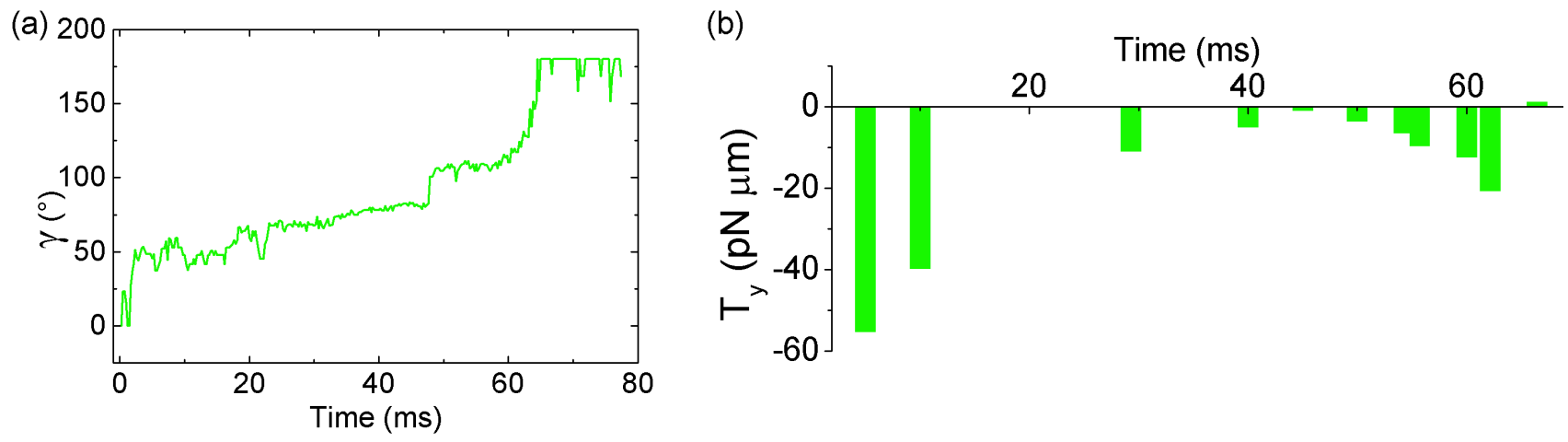

Figure 7. (a) Time evolution of the peanut-shaped particle angle $\gamma$ measured experimentally and (b) simulated optical torque acting on the particle along the $\mathrm{Y}$-axis $\left(\mathrm{T}_{\mathrm{y}}\right)$. In the simulations, the particle is considered closer to one beam during the first part of the measurement $(t \in[0,48] \mathrm{ms})$ and then closer to the second beam during the second part of the data $(\mathrm{t} \epsilon[48,77] \mathrm{ms})$.

As shown by Figure 8-a, the angle $\alpha$ of the peanut-shaped particle remains around 2-3 $3^{\circ}$ from $t=0$ up to 18 ms. $\alpha$ then increases almost linearly up to $9^{\circ}$ at $t=46 \mathrm{~ms}$. After $\mathrm{t}=48 \mathrm{~ms}$ when the particles flips to $\gamma>90^{\circ}, \alpha$ rapidly becomes 
negative and reaches a minimum of $\sim-6^{\circ}$ before reaching values around $-1^{\circ}$ to $-2^{\circ}$ as the particles aligns in the reconstruction plane. Qualitatively the simulated torque acting along the $\mathrm{X}$-axis $\left(\mathrm{T}_{\mathrm{x}}\right)$ is in very good agreement with the observed $\alpha . T_{x}$ is increasing from $t=0$ to $45 \mathrm{~ms}$, before becoming negative. The absolute value of $T_{x}$ then decreases until $\mathrm{t}=62 \mathrm{~ms}$. The higher absolute value of the torque in the first part of the simulation also predicts a higher $\alpha$ during this part than in the second part of the measurement.
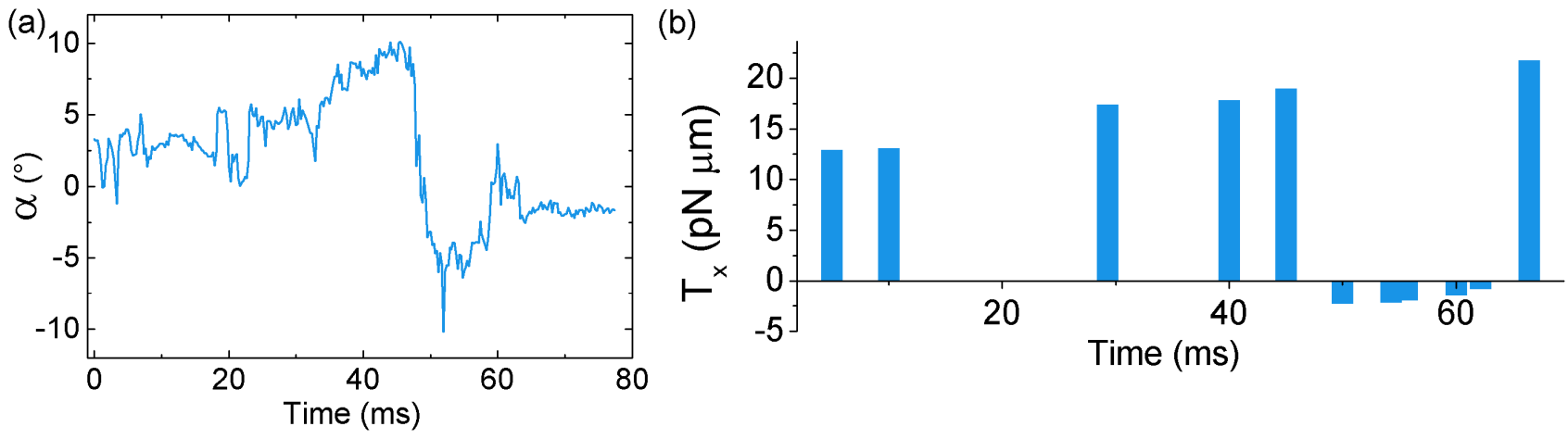

Figure 8. (a) Time evolution of the peanut-shaped particle angle $\alpha$ measured experimentally and (b) simulated optical torque acting on the particle along the $\mathrm{X}$-axis $\left(\mathrm{T}_{\mathrm{x}}\right)$. In the simulations, the particle is considered closer to one beam during the first part of the measurement $(\mathrm{t} \epsilon[0,48] \mathrm{ms})$ and then closer to the second beam during the second part of the data $(\mathrm{t} \epsilon[48,77] \mathrm{ms})$.

The previous figures present separately the particle motion through different degrees of freedom. Comparison between these degrees of freedom reveals some coupling between the translational and rotational motions. For instance the $\mathrm{X}$ position of the particle appears to be anti-correlated with the orientation angle $\alpha$. In addition, the rotation angle $\gamma$ and the particle translation along $\mathrm{Z}$ also seem to be anti-correlated. After $\mathrm{t}=64 \mathrm{~ms}$, both $\mathrm{Z}$ and $\gamma$ barely change, but they also both change very quickly between 57 and $64 \mathrm{~ms}$.

\section{DISCUSSION}

The digital holography of optically-trapped aerosol particle opens up new possibilities for experiments. We show here how such measurements can be used to retrieve the 6D motion of nonspherical particles. FDTD calculations of optical forces and torques are compared with the experimental motion of a peanut-shaped particle in the trap. The main general features of the particle dynamics are in a good agreement with the simulated optical forces and torques. For example, the rotation of the particle (angles $\gamma$ and $\alpha$ shown in Figure 7 and 8) are in good qualitative agreement with the experiment. The simulations also show that the particle is closer to one trapping beam during the first part of the measurement and closer to the second beam during the second part of the measurements. However, some features of the particle motion could not be explained by our calculations. For example, the fluctuations of the particle velocity along the Z-axis cannot be explained by our calculations, probably because the real trap alignment is not as simple as assumed in the calculations. Nonetheless, comparing the measured motion of trapped nonspherical particles with calculations helps to better understand the trapping of nonspherical aerosol particles and the coupling between their rotational and translational motion. Our experiment is currently being coupled with broadband light scattering (BLS) to elucidate the mechanism of phase transitions of aerosol particles, which can proceed through multiple pathways including multistep processes $^{48}$. Measuring the morphology changes of the particles during their phase transitions with high time and spatial resolution will help to shed some light on the intriguing details of such mechanisms, which currently lie largely in the dark. 


\section{ACKNOWLEDGMENTS}

This work was supported by the Swiss National Science Foundation (SNSF grant no. 200020_172472), ETH Zurich and an ETH Career Seed Grant SEED-67 16-1. We would like to thank David Stapfer and Markus Steger from the ETH mechanical and electronic shop for their help.

\section{REFERENCES}

[1] Ashkin, A. and Dziedzic, J. M. Observation of light scattering from nonspherical particles usingoptical levitation. Appl. Opt. 19, 660-668 (1980).

[2] Buajarern, J., Mitchem, L., Ward, A. D., Nahler, N. H., McGloin, D. and Reid, J. P. Controlling and characterizing the coagulation of liquid aerosol droplets. J. Chem. Phys. 125, 114506 (2006).

[3] Bui, A. A. M., Stilgoe, A. B., Nieminen, T. A. and Rubinsztein-Dunlop, H. Calibration of nonspherical particles in optical tweezers using only position measurement. Opt. Lett. 38, 1244-1246 (2013).

[4] Cao, Y., Stilgoe, A. B., Chen, L., Nieminen, T. A. and Rubinsztein-Dunlop, H. Equilibrium orientations and positions of non-spherical particles in optical traps. Opt. Express. 20, 12987-12996 (2012).

[5] Zheng, F. Thermophoresis of spherical and non-spherical particles: a review of theories and experiments. $A d v$. Colloid Interface Sci. 97, 255-278 (2002).

[6] Burns, M. M., Fournier, J.-M. and Golovchenko, J. A. Optical binding. Physical Review Letters. 63, 1233-1236 (1989).

[7] Karásek, V., Čižmár, T., Brzobohatý, O., Zemánek, P., Garcés-Chávez, V. and Dholakia, K. Long-Range OneDimensional Longitudinal Optical Binding. Physical Review Letters. 101, 143601 (2008).

[8] Thanopulos, I., Luckhaus, D. and Signorell, R. Modeling of optical binding of submicron aerosol particles in counterpropagating Bessel beams. Phys. Rev. A. 95, 063813 (2017).

[9] Zhang, R., Khalizov, A. F., Pagels, J., Zhang, D., Xue, H. and McMurry, P. H. Variability in morphology, hygroscopicity, and optical properties of soot aerosols during atmospheric processing. Proc. Natl. Acad. Sci. USA. 105, 10291-10296 (2008).

[10] Möhler, O., Field, P. R., Connolly, P., Benz, S., Saathoff, H., Schnaiter, M., Wagner, R., Cotton, R., Krämer, M., Mangold, A. and Heymsfield, A. J. Efficiency of the deposition mode ice nucleation on mineral dust particles. Atmos. Chem. Phys. 6, 3007-3021 (2006).

[11] Vortisch, H., Kramer, B., Weidinger, I., Woste, L., Leisner, T., Schwell, M., Baumgartel, H. and Ruhl, E. Homogeneous freezing nucleation rates and crystallization dynamics of single levitated sulfuric acid solution droplets. Phys. Chem. Chem. Phys. 2, 1407-1413 (2000).

[12] Colberg, C. A., Krieger, U. K. and Peter, T. Morphological Investigations of Single Levitated H2SO4/NH3/H2O Aerosol Particles during Deliquescence/Efflorescence Experiments. J. Phys. Chem. A. 108, 27002709 (2004).

[13] Lu, J. W., Isenor, M., Chasovskikh, E., Stapfer, D. and Signorell, R. Low-temperature Bessel beam trap for single submicrometer aerosol particle studies. Rev. Sci. Instrum. 85, 095107 (2014).

[14] Cammenga, H. K., Schulze, F. W. and Theuerl, W. Vapor pressure and evaporation coefficient of glycerol. $J$. Chem. Eng. Data. 22, 131-134 (1977).

[15] Laskin, A., Laskin, J. and Nizkorodov, S. A. Chemistry of Atmospheric Brown Carbon. Chem. Rev. 115, 43354382 (2015).

[16] Cwiertny, D. M., Young, M. A. and Grassian, V. H. Chemistry and Photochemistry of Mineral Dust Aerosol. Annu. Rev. Phys. Chem. 59, 27-51 (2008).

[17] Dupart, Y., King, S. M., Nekat, B., Nowak, A., Wiedensohler, A., Herrmann, H., David, G., Thomas, B., Miffre, A., Rairoux, P., D'Anna, B. and George, C. Mineral dust photochemistry induces nucleation events in the presence of SO2. Proc. Natl. Acad. Sci. USA. 109, 20842-20847 (2012).

[18] Bohren, C. F. and Huffman, D. R., Absorption and Scattering of Light by Small Particles (Wiley, 1983).

[19] Mishchenko, M. I., Travis, L. D. and Lacis, A. A., Scattering, Absorption, and Emission of Light by Small Particles (Cambridge university press, 2002). 
[20] Moosmüller, H., Chakrabarty, R. K. and Arnott, W. P. Aerosol light absorption and its measurement: A review. J. Quant. Spectrosc. Radiat. Transfer. 110, 844-878 (2009).

[21] Shemesh, D. and Gerber, R. B. Femtosecond timescale deactivation of electronically excited peroxides at ice surfaces. Mol. Phys. 110, 605-617 (2012).

[22] Kaufman, Y. J., Tanre, D. and Boucher, O. A satellite view of aerosols in the climate system. Nature. 419, 215 223 (2002).

[23] IPCC 2014: Climate Change 2014: Synthesis Report. Contribution of Working Groups I, II and III to the Fifth Assessment Report of the Intergovernmental Panel on Climate Change [Core Writing Team, R.K. Pachauri and L.A. Meyer (eds.)]. IPCC, Geneva, Switzerland, 151 pp. Journal. (2014).

[24] Gage, S. H. Modern Dark-Field Microscopy and the History of Its Development. Transactions. 39, 95-141 (1920).

[25] David, G., Esat, K., Thanopulos, I. and Signorell, R. Digital holography of optically-trapped aerosol particles. Communications Chemistry. (2018, accepted).

[26] Horstmeyer, R., Chung, J., Ou, X., Zheng, G. and Yang, C. Diffraction tomography with Fourier ptychography. Optica. 3, 827-835 (2016).

[27] Tian, L., Liu, Z., Yeh, L.-H., Chen, M., Zhong, J. and Waller, L. Computational illumination for high-speed in vitro Fourier ptychographic microscopy. Optica. 2, 904-911 (2015).

[28] Choi, W. Tomographic phase microscopy and its biological applications. 3D Research. 3, 5 (2012).

[29] Jeong, H.-j., Yoo, H. and Gweon, D. High-speed 3-D measurement with a large field of view based on directview confocal microscope with an electrically tunable lens. Opt. Express. 24, 3806-3816 (2016).

[30] Xu, W., Jericho, M., Meinertzhagen, I. and Kreuzer, H. Digital in-line holography for biological applications. Proc. Natl. Acad. Sci. USA. 98, 11301-11305 (2001).

[31] Kemppinen, O., Heinson, Y. and Berg, M. Quasi-three-dimensional particle imaging with digital holography. Appl. Opt. 56, F53-F60 (2017).

[32] Javidi, B. and Kim, D. Three-dimensional-object recognition by use of single-exposure on-axis digital holography. Opt. Lett. 30, 236-238 (2005).

[33] Prodi, F., Santachiara, G., Travaini, S., Belosi, F., Vedernikov, A., Dubois, F., Queeckers, P. and Legros, J. C. Digital holography for observing aerosol particles undergoing Brownian motion in microgravity conditions. Atmospheric Research. 82, 379-384 (2006).

[34] Garcia-Sucerquia, J., Xu, W., Jericho, S. K., Klages, P., Jericho, M. H. and Kreuzer, H. J. Digital in-line holographic microscopy. Appl. Opt. 45, 836-850 (2006).

[35] Wang, A., Dimiduk, T. G., Fung, J., Razavi, S., Kretzschmar, I., Chaudhary, K. and Manoharan, V. N. Using the discrete dipole approximation and holographic microscopy to measure rotational dynamics of non-spherical colloidal particles. J. Quant. Spectrosc. Radiat. Transfer. 146, 499-509 (2014).

[36] Chiong Cheong, F. and Grier, D. G. Rotational and translational diffusion of copper oxide nanorods measured with holographic video microscopy. Opt. Express. 18, 6555-6562 (2010).

[37] Berg, M. J. and Videen, G. Digital holographic imaging of aerosol particles in flight. J. Quant. Spectrosc. Radiat. Transfer. 112, 1776-1783 (2011).

[38] Jericho, S. K., Garcia-Sucerquia, J., Xu, W., Jericho, M. H. and Kreuzer, H. J. Submersible digital in-line holographic microscope. Rev. Sci. Instrum. 77, 043706 (2006).

[39] Miles, R. E. H., Carruthers, A. E. and Reid, J. P. Novel optical techniques for measurements of light extinction, scattering and absorption by single aerosol particles. Laser Photon. Rev. 5, 534-552 (2011).

[40] Butler, J. R., Mitchem, L., Hanford, K. L., Treuel, L. and Reid, J. P. In situ comparative measurements of the properties of aerosol droplets of different chemical composition. Faraday Discuss. 137, 351-366 (2008).

[41] King, M. D., Thompson, K. C. and Ward, A. D. Laser Tweezers Raman Study of Optically Trapped Aerosol Droplets of Seawater and Oleic Acid Reacting with Ozone: Implications for Cloud-Droplet Properties. J. Am. Chem. Soc. 126, 16710-16711 (2004).

[42] Cremer, J. W., Thaler, K. M., Haisch, C. and Signorell, R. Photoacoustics of single laser-trapped nanodroplets for the direct observation of nanofocusing in aerosol photokinetics. Nat. Commun. 7, 10941 (2016).

[43] David, G., Esat, K., Ritsch, I. and Signorell, R. Ultraviolet broadband light scattering for optically-trapped submicron-sized aerosol particles. Phys. Chem. Chem. Phys. 18, 5477-5485 (2016).

[44] Lee, A. K. Y., Ling, T. Y. and Chan, C. K. Understanding hygroscopic growth and phase transformation of aerosols using single particle Raman spectroscopy in an electrodynamic balance. Faraday Discuss. 137, 245-263 (2008). 
[45] Esat, K., David, G., Poulkas, T., Shein, M. and Signorell, R. Phase transition dynamics of single optically trapped aqueous potassium carbonate particles. Phys. Chem. Chem. Phys. (2018).

[46] Li, T., Kheifets, S., Medellin, D. and Raizen, M. G. Measurement of the Instantaneous Velocity of a Brownian Particle. Science. 328, 1673-1675 (2010).

[47] Rkiouak, L., Tang, M. J., Camp, J. C. J., McGregor, J., Watson, I. M., Cox, R. A., Kalberer, M., Ward, A. D. and Pope, F. D. Optical trapping and Raman spectroscopy of solid particles. Phys. Chem. Chem. Phys. 16, 11426-11434 (2014).

[48] Esat, K., David, G., Poulkas, T., Shein, M. and Signorell, R. Phase transition dynamics of single optically trapped aqueous potassium carbonate particles. Phys. Chem. Chem. Phys. 20, 11598-11607 (2018).

[49] Jackson, J. D. Classical Electrodynamics John Wiley \& Sons. Inc., New York. (1999).

[50] Taflove, A. and Hagness, S. C., Computational electrodynamics: the finite-difference time-domain method (Artech House, 2005).

[51] Sullivan, D. M., Electromagnetic simulation using the FDTD method (John Wiley \& Sons, 2013).

[52] See www.lumerical.com for FDTD Solutions, V., Lumerical Solutions, Inc. 